\author{
Elsayed Abo-salem*, Mouhamad \\ Abdallah, Mohamed Effat, Said \\ Alsidawi and Myron Gerson \\ University of Cincinnati, Department of \\ cardiovascular health and diseases, Cincinnati, $\mathrm{OH}$ \\ USA \\ Dates: Received: 06 March, 2016; Accepted: 13 \\ April, 2016; Published: 15 April, 2016 \\ *Corresponding author: Elsayed Abo-salem, MD, \\ Division of Cardiovascular Diseases, 231 Albert Sabin \\ Way, Room 3461 MSB, PO Box: 670542, Cincinnati, \\ $\mathrm{OH}, 45267-0542$, USA, Tel: 513-558-4231; Fax: 513- \\ 558-0852; E-mail: abosaled@ucmail.uc.edu \\ www.peertechz.com
}

\section{Research Article \\ Correlation of Cardiac Sympathetic Nervous System Dysfunction with Diastolic Left Ventricular Dysfunction in Patients with Controlled Hypertension}

\section{Abbreviations}

${ }^{123} \mathrm{I}$ mIBG: ${ }^{123}$ I-metaiodobenzylguanidine; H/M: Heart to Mediastinum ratio; WOR: Washout Rate; NE: Norepinephrine; ACE: Angiotensin Converting Enzyme; ARB: Angiotensin Receptor blocker.

\section{Introduction}

${ }^{123} \mathrm{I}$-metaiodobenzylguanidine (mIBG) is a radioactive analogue of norepinephrine (NE) up taken at the sympathetic nerve ending and can be imaged with planar or Single-photon emission computed tomography images. A reduction of the ${ }^{123} \mathrm{I}-\mathrm{MIBG}$ uptake by the nerve ending in the heart compared to mediastinum (heart to mediastinum ratio $[\mathrm{H} / \mathrm{M}])$ is a marker of localized cardiac autonomic function. Subjects with hypertension have a lower ${ }^{123} \mathrm{I}-\mathrm{MIBG}$ uptake and H/M ratio, and a higher washout rate (WOR) compared to subjects with normal blood pressure [1]. In a previous report by Kuwahara T et al., left ventricular mass index had a negative correlation with $\mathrm{H} / \mathrm{M}$ ratio on both early and delayed images, and a positive correlation with the WOR [2]. Serum NE level is also elevated in subjects with essential hypertension compared to normal blood pressure, and positively correlates with left ventricular muscle mass index and wall thickness $[3,4]$.

Cardiac autonomic dysfunction plays a pivotal role in the pathogenesis of heart failure with reduced left ventricular ejection fraction. Angiotensin II and a1-adrenergic receptor agonist also induce myocardial hypertrophy and diastolic dysfunction in animal models [5]. The role of sympathetic dysfunction in the pathophysiology of diastolic dysfunction without heart failure among subjects treated with angiotensin converting enzyme (ACE) inhibitors or angiotensin receptor blockers (ARBs) is unknown. Among subjects with hypertension controlled with ACE inhibitors or ARBs, our hypothesis suggests a lower ${ }^{123}$ I-MIBG uptake and H/M ratio, and a higher washout rate (WOR) in cases with grade I diastolic dysfunction compared to cases with normal diastolic function.

\section{Patients and Methods}

\section{Patients}

The study was approved by the Institutional Review Boards/Ethics 
Committees in the University of Cincinnati, OH (Protocol number; 07011201). Informed consent was obtained from the patients. The study design is prospective and enrolled 24 outpatients, 20 to 80 years of age and both genders, with well-controlled hypertension. Wellcontrolled hypertension was defined as a systolic blood pressure less than $140 \mathrm{mmHg}$ and diastolic blood pressure less than $90 \mathrm{mmHg}$ on study entry. Patients were on stable antihypertensive regimen including an ACE inhibitor or ARBs for at least 2 months prior to enrollment.

Patients were excluded if they had any of the following: 1) known valvular heart disease of more than mild severity, 2) known coronary artery disease defined by an angiographic coronary artery stenosis greater than or equal to $50 \%$ luminal diameter narrowing, acute or previous myocardial infarction, or previous coronary revascularization, 3) left ventricular ejection fraction less than $50 \%, 4$ ) atrial fibrillation, 5) current treatment with a $\beta$-adrenergic blocking drug, diltiazem or verapamil 6) current treatment with a psychoactive or other drug known to alter ${ }^{123}$ I-MIBG uptake, 7) participation in another research study within the prior 30 days, 8) pregnancy or breast feeding, 9) inability or unwillingness to provide informed consent, 10) diabetes mellitus on medical therapy, 11) iodine allergy

Ambulatory blood pressure monitoring was used to record accurate measurements of systolic, diastolic, and mean blood pressures and quantitation of the nocturnal blood pressure dip. ${ }^{123} \mathrm{I}$ mIBG imaging was performed on all study patients to determine heart to mediastinum $(\mathrm{H} / \mathrm{M})$ ratio. A ten minute planar image over the anterior chest was obtained beginning at 15 and 240 minutes after injection of $5 \mathrm{mCi}$ of ${ }^{123} \mathrm{I}$ mIBG. Images were acquired using a dual head Siemens camera with a $159 \mathrm{keV}$ symmetric $20 \%$ window. Images were stored in a $64 \times 64$ matrix, with use of camera zoom factor as appropriate to achieve a normal pixel size of $6.4 \mathrm{~mm}$ (range 6.0 to 6.8). The H/M ratio was measured at 15 minutes and 230 minutes using the ratio of heart counts per pixel to mediastinal counts per pixel without background subtraction.

All echocardiograms were performed on Philips ATL HDI 5000 (Serial\# 01414Q). Data were digitally analyzed offline on Camtronics Vericis Software at echo reading workstations. Left ventricular diastolic function was comprehensively assessed using a combination of 2D echo and Doppler indices. Early (E) and late mitral inflow (A) diastolic velocities, E/A ratio, deceleration time and isovolumetric relaxation time were obtained from conventional spectral Doppler. Tissue Doppler of the lateral mitral annulus was used to assess early diastolic annular velocity (Ea). E/Ea ratio was used to assess filling pressures. Echocardiograms were read by an independent echocardiographer. Patients were classified as having normal or abnormal diastolic function -according to American Society of Echocardiography guidelines- based on mitral inflow Doppler, tissue Doppler velocity of the lateral and septal mitral annulus and pulmonary vein Doppler flow. Only cases with normal or grade I diastolic dysfunction were included, while cases with grade II, III or IV diastolic dysfunction and elevated filling pressures were excluded.

The echocardiograms and nuclear images were interpreted by independent operators who were blinded to the results of other studies. Blood samples were collected for measurement of plasma NE $(\mathrm{ng} / \mathrm{ml})$ and angiotensin II levels (pg/ml). Patients were rested in a quiet dark room for 30 minutes prior to sample collection. All blood samples were collected on ice, centrifuged at 4 degrees Celsius, and stored frozen. Samples were sent to an independent laboratory for analysis.

\section{Statistical analysis}

Continuous data were reported as means and standard deviations, and unpaired $t$ tests were used for 2 group comparisons. Data that didn't have a normal distribution were reported as medians and compared with Mann-Whitney statistical tests for 2 groups. Nominal data were expressed as percentages and numbers, and analysis was performed by Chi-square test or Fisher's exact test for sample sizes less than 5 . A probability value $<0.05$ identified a statistically significant result. Linear regression analysis was used to study the degree of relation between diastolic dysfunction (as measured by E/A ratio) with degree of sympathetic nervous system dysfunction as measured by $\mathrm{H} / \mathrm{M}$ ratio and plasma norepinephrine level. Correlation analysis was performed between plasma norepinephrine and $\mathrm{H} / \mathrm{M}$ ratio and WOR. Absolute values of r, less than $0.4,0.4$ to 0.6 and more than 0.6 were considered as weak, moderate, and strong, respectively. Statistical analyses were performed using MedCalc, version 12.5 (MedCalc Software, Ostend, Belgium)

\section{Results}

Patients were divided into two groups; group A (grade I diastolic dysfunction, 10 patients) and group B (normal diastolic function, 14 patients). There was no difference between both groups on the baseline characteristics (Table 1). Group A included 10 subjects with grade I diastolic dysfunction but no echocardiographic criteria for elevated left atrium filling pressure. None of the patients had a clinical diagnosis of heart failure or structural heart disease other than left ventricular hypertrophy. The prevalence of co-morbid conditions among study subjects was low and none of the subjects had an estimated glomerular filtration rate less than $60 \mathrm{ml} / \mathrm{min}$. The blood pressure was controlled with ACE inhibitors in 22 subjects or ARBs in 2 subjects. There were no significant differences among groups in regard to serum angiotensin II level (Table 1). The degree of blood pressure control was equal as shown by ambulatory blood pressure monitoring, with no significant difference in the systolic or diastolic blood pressure or nocturnal dip in blood pressure (Table 1).

There were no significant differences between study groups with regard to early ${ }^{123}$ I-MIBG activity or WOR (Table 1 ). Patients with diastolic dysfunction had a higher level of plasma NE ( $0.46 \mathrm{vs} 0.26 \mathrm{ng}$ / $\mathrm{ml}, \mathrm{p}=0.01$ ) as compared to patients with normal diastolic function (Table 1). There was a statistically significant inverse correlation between $\mathrm{E} / \mathrm{A}$ ratio and the $\log$ of plasma norepinephrine level $(\mathrm{r}=-0.43, \mathrm{P}=0.03)$ (Figure 1$)$. There was no statistically significant correlation between $\mathrm{E} / \mathrm{A}$ ratio and early $\mathrm{H} / \mathrm{M}$ ratio $(\mathrm{p}=0.07)$ (Figure $2)$, late $\mathrm{H} / \mathrm{M}$ ratio $(\mathrm{p}=0.12)$ or WOR $(\mathrm{p}=0.99)$. There was also no correlation between plasma NE and early or late $\mathrm{H} / \mathrm{M}$ ratio or WOR $(\mathrm{p}=0.85, \mathrm{p}=0.89, \mathrm{p}=0.52)$.

\section{Discussion}

In this study, the level of cardiac sympathetic nervous system 
Table 1: Baseline characteristics and sympathetic system activity in subjects with normal and abnormal diastolic function.

\begin{tabular}{|c|c|c|c|}
\hline & $\begin{array}{l}\text { Diastolic } \\
\text { dysfunction } \\
(10)\end{array}$ & $\begin{array}{l}\text { Normal diastolic } \\
\text { function (14) }\end{array}$ & $P$ value \\
\hline \multicolumn{4}{|l|}{ Baseline characteristics } \\
\hline Age (years) & $61.5 \pm 6$ & $58.9 \pm 11$ & 0.5 \\
\hline Gender (F) \% & $50 \%$ & $64.3 \%$ & 0.8 \\
\hline Body mass index (kg/m2) & $31.7 \pm 8$ & $29.8 \pm 9$ & 0.6 \\
\hline \multicolumn{4}{|l|}{ Antihypertensive medications } \\
\hline ACEls, $n$ & 8 & 14 & 0.16 \\
\hline ARBs, $n$ & 2 & 0 & 0.18 \\
\hline Amlodipine, $\mathrm{n}$ & 2 & 0 & 0.18 \\
\hline Hydrochlorothiazide, $\mathrm{n}$ & 1 & 1 & 1 \\
\hline \multicolumn{4}{|l|}{ Echocardiography } \\
\hline Mitral E wave (cm/sec) & $61.9 \pm 8$ & $79.6 \pm 11$ & $<0.001$ \\
\hline Mitral E/A ratio (median) & 0.83 & 1.22 & $<0.001$ \\
\hline Lateral MA é velocity (cm/sec) & $9.8 \pm 3$ & $13.4 \pm 4$ & 0.01 \\
\hline E/é ratio & $7 \pm 2$ & $6.2 \pm 2$ & 0.3 \\
\hline Deceleration time (milliseconds) & $251 \pm 22$ & $194 \pm 26$ & $<0.001$ \\
\hline IVRT (milliseconds) & $115 \pm 19$ & $95 \pm 16$ & 0.01 \\
\hline Left atrium area $(\mathrm{cm} 2)$ & $17.9 \pm 2.92$ & $17.5 \pm 3.13$ & 0.7 \\
\hline Left atrium volume (cm3) & $46.11 \pm 15.39$ & $43.43 \pm 10.70$ & 0.6 \\
\hline Interventricular septum (cm) & $1.14 \pm 0.19$ & $1.14 \pm 0.27$ & 1 \\
\hline Posterior wall thickness (cm) & $1.17 \pm 0.17$ & $1.21 \pm 0.23$ & 0.7 \\
\hline \multicolumn{4}{|l|}{ 123I-mIBG scan } \\
\hline H/M 15 minutes & $1.6 \pm 0.2$ & $1.7 \pm 0.2$ & 0.6 \\
\hline H/M 240 minutes & $1.6 \pm 0.2$ & $1.6 \pm 0.2$ & 0.9 \\
\hline Wash out ratio \% & $3.1 \pm 8.7$ & $6.4 \pm 8.9$ & 0.4 \\
\hline \multicolumn{4}{|l|}{ Laboratory data } \\
\hline Plasma NE (ng/ml), median & 0.46 & 0.26 & 0.02 \\
\hline Angiotensin II (pg/ml), median & 12.5 & 7.7 & 0.4 \\
\hline \multicolumn{4}{|c|}{ Ambulatory blood pressure monitoring } \\
\hline Systolic BP (mmHg) & $124.1 \pm 10.7$ & $125.3 \pm 13$ & 0.8 \\
\hline Diastolic BP (mmHg) & $72.7 \pm 6.8$ & $74 \pm 10$ & 0.7 \\
\hline Systolic BP dip (mmHg) & $10.5 \pm 11$ & $11.8 \pm 12$ & 0.8 \\
\hline
\end{tabular}

A probability value $<0.05$ identified a statistically significant result.

ACEI: Angiotensin Converting Enzyme Inhibitor, ARBs: Angiotensin Receptors Blockers; MA: Mitral Annulus; IVRT: Isovolumetric Relaxation Time, H/M: Heart Mediastinum Ratio, NE: Norepinephrine, BP: Blood Pressure;

All value are reported as mean \pm standard deviation unless specified.

dysfunction measured by ${ }^{123} \mathrm{I}$ mIBG $\mathrm{H} / \mathrm{M}$ ratio did not differ significantly in patients with and without left ventricular diastolic dysfunction ( 1.68 vs 1.63 , respectively, $\mathrm{p}=0.57$ ). Additionally the $\mathrm{H} / \mathrm{M}$ ratio did not correlate significantly with individual indices of diastolic dysfunction (E/A ratio, isovolumetric relaxation time, and deceleration time). Localized cardiac sympathetic nervous system dysfunction was not a major determinant of early diastolic dysfunction in the present study population with well controlled hypertension using ACE inhibitors. Alternative central or peripheral mechanisms may have had a more significant role in the pathogenesis of diastolic function in these cases.

All the patients in our study were using either ACE inhibiter or ARB. Several previous studies of patient with heart failure and left ventricular systolic dysfunction have confirmed an improved ${ }^{123}$ I-MIBG uptake with ACE inhibitors [6,7]. In a double-blind randomized controlled study with candesartan versus placebo, 50 patients with congestive heart failure and preserved ejection fraction underwent ${ }^{123}$ I-MIBG scan at baseline and after 6 months of therapy. The $\mathrm{H} / \mathrm{M}$ ratio increased $(1.87$ to $2, \mathrm{P}<0.005)$ and ${ }^{123} \mathrm{I}$-MIBG WOR decreased ( 37 to $32 \%, \mathrm{P}<0.005$ ) with candesartan after 6 months of therapy [6]. The reduction in the left ventricular mass with blood pressure control is associated with an increase in the $\mathrm{H} / \mathrm{M}$ ratio and decrease in WOR [8].

Plasma NE level was significantly elevated in our subjects with diastolic dysfunction compared to subjects with normal cardiac relaxation, despite the use of ACE inhibitor or ARB and adequate blood pressure control in ambulatory monitoring. In a study of subjects with hypertension and diastolic dysfunction, the sympathetic nerve activity was significantly elevated compared to hypertensive controls without diastolic dysfunction [9]. There was also a moderate inverse correlation between plasma NE level and E/A ratio in our study. However, it is uncertain whether the higher plasma NE level is a cause, consequence, or association with diastolic dysfunction. Left atrium was also not dilated, left ventricular filling pressures were normal (grade I diastolic dysfunction with a normal E/é ratio), and none of the patients had not been diagnosed with clinical heart failure at the time of enrollment. These findings suggest that plasma NE is unlikely secondary to diastolic heart failure.

ACE inhibitors did not appear to result in an absolute reduction in the plasma NE level in other trials [10]. In a study of 24 patients with congestive heart failure, benazepril reduced sympathetic nerve trafficking, though plasma NE level was not significantly altered [10]. The correlation between early or late $\mathrm{H} / \mathrm{M}$ ratio and plasma NE level was also weak in a study of 35 patients with ischemic cardiomyopathy [11]. In another study of 23 patients with reduced left ventricular systolic function, ${ }^{123} \mathrm{I}-\mathrm{mIBG}$ scan was performed at baseline and 6 weeks after treatment with enalapril. Cardiac uptake of ${ }^{123} \mathrm{I}-\mathrm{mIBG}$ increased significantly after treatment with enalapril, despite no significant changes in the serum NE level [12]. All the subjects in our study were on ACE inhibitors or ARBs for at least 2 months before enrollment and this may have contributed to the increase in ${ }^{123} \mathrm{I}-\mathrm{mIBG}$ without a reduction in plasma NE level.

The reason for the lack of correlation between the plasma NE and $\mathrm{H} / \mathrm{M}$ ratio is uncertain. Angiotensin II stimulates the release of NE from the adrenal gland; and secretion can decrease with ACE inhibitors or ARBs [13]. However, bradykinin, that increases with ACE inhibitors or ARBs treatment, stimulates the release of cathecholamines from the adrenal gland [14]. Plasma NE level is also affected by the rate of presynaptic reuptake and tissue clearance. Treatment with ACE inhibitors is known to increase the NE uptake at the presynaptic nerve [15], which may explain an increase in $\mathrm{H} / \mathrm{M}$ ratio. However, ACE inhibitors do not decrease the rate of 


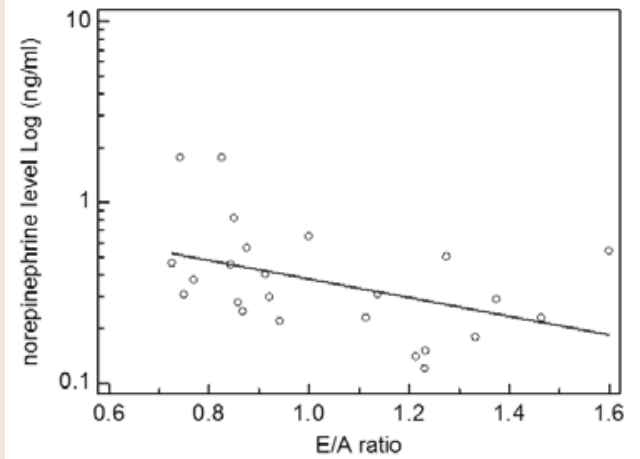

Figure 1: Linear regression analysis for the relation between E/A ratio and plasma norepinephrine log $(\mathrm{ng} / \mathrm{ml})$.

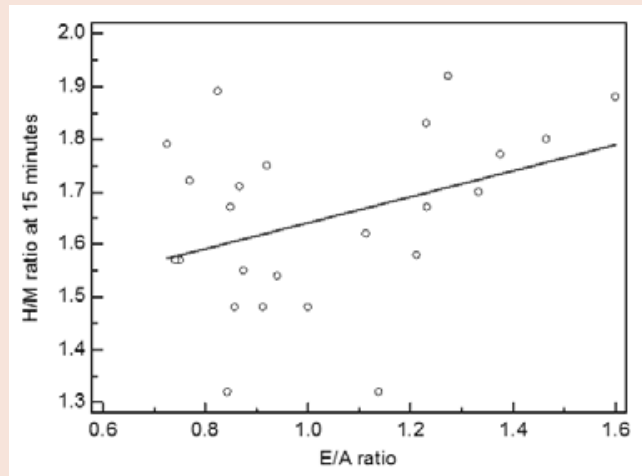

Figure 2: Linear regression analysis for the relation between E/A ratio and early heart mediastinum $(\mathrm{H} / \mathrm{M})$ ratio.

catecholamine release significantly particularly with bradykinin accumulation that increases NE release [16].

All the subjects in our study with abnormal diastolic function were a grade I diastolic dysfunction with no other identified cardiac pathology. Blood pressure was also well-controlled with a single antihypertensive agent in most patients. This may limit our conclusions to subjects with a milder form of hypertensive heart disease and early stages of diastolic dysfunction. Cardiac sympathetic dysfunction may play a significant role in cases with more advanced degrees of diastolic dysfunction. Studies that primarily included subjects with heart failure showed a significant correlation between the echocardiographic parameters of diastolic dysfunction and H/M ratio, particularly among subjects with an elevated left atrium pressure [17]. These correlations were not significant in our subjects with grade I diastolic dysfunction with no echocardiographic signs of elevated left atrial pressure.

Limitations for the study include a small sample size that may have limited the statistical power. Most of the subjects in our study received ACE inhibitors and corresponding conclusions with ARBs may not be applicable. The duration of hypertension or treatment with ACE inhibitors or ARB was not recorded and may have a potential influence of therapy on the cardiac autonomic dysfunction and progression to diastolic dysfunction. The limitations of serum
$\mathrm{NE}$ as a marker of sympathetic nervous system activation should also be taken into account. Plasma levels can also vary significantly and acutely with psychological stress, caffeine intake and medications. The contribution of autonomic dysfunction in the pathogenesis of heart disease may also vary among different ethnicities or age groups. Most of our patients were Caucasian males. There was slightly more males in the diastolic dysfunction group (50\%) compared to normal subjects (36\%), though not statistically significant. Baseline sympathetic activity is higher in males than females and response to physiological and pathological stressors may be different [18]. A follow up was not available to identify the prognosis among our subjects including the progression of hypertensive heart disease. The study of cardiac sympathetic activity can be challenging due to influence and interaction from peripheral and central autonomic control [19]. Carotid body chemoreceptors may also play a role in the progression of heart failure [20].

\section{Conclusion}

Localized cardiac autonomic dysfunction is not significantly worse in grade I diastolic dysfunction, compared to normal diastolic function, in patients with systemic hypertension that is well-controlled on ACE inhibitors, despite a significant elevation in plasma norepinephrine level with grade I diastolic dysfunction.

\section{References}

1. Sakata K, Shirotani M, Yoshida H, Kurata C (1999) Cardiac sympathetic nervous system in early essential hypertension assessed by 123I-MIBG. J Nucl Med 40: 6-11.

2. Kuwahara T, Hamada M, Hiwada K (1998) Direct evidence of impaired cardiac sympathetic innervation in essential hypertensive patients with left ventricular hypertrophy. J Nucl Med 39: 1486-1491.

3. Corea L, Bentivoglio M, Verdecchia P (1983) Echocardiographic left ventricular hypertrophy as related to arterial pressure and plasma norepinephrine concentration in arterial hypertension. Reversal by atenolol treatment. Hypertension 5: 837-843.

4. Burns J, Sivananthan MU, Ball SG, Mackintosh AF, Mary DA, et al. (2007) Relationship between central sympathetic drive and magnetic resonance imaging-determined left ventricular mass in essential hypertension. Circulation 115: 1999-2005.

5. Mori J, Basu R, McLean BA, Das SK, Zhang L, et al. (2012) Agonist-induced hypertrophy and diastolic dysfunction are associated with selective reduction in glucose oxidation: a metabolic contribution to heart failure with normal ejection fraction. Circ Heart Fail 5: 493-503.

6. Kasama S, Toyama T, Kumakura H, Takayama Y, Ichikawa S, et al. (2005) Effects of candesartan on cardiac sympathetic nerve activity in patients with congestive heart failure and preserved left ventricular ejection fraction. J Am Coll Cardiol 45: 661-667.

7. Sakata K, Shirotani M, Yoshida H, Kurata C (1998) Comparison of effects of enalapril and nitrendipine on cardiac sympathetic nervous system in essential hypertension. J Am Coll Cardiol 32: 438-443.

8. Morimoto S, Terada K, Keira N, Satoda M, Inoue K, et al. (1996) Investigation of the relationship between regression of hypertensive cardiac hypertrophy and improvement of cardiac sympathetic nervous dysfunction using iodine-123 metaiodobenzylguanidine myocardial imaging. Eur J Nucl Med 23: $756-761$.

9. Grassi G, Seravalle G, Quarti-Trevano F, Dell'Oro R, Arenare F, et al. (2009) Sympathetic and baroreflex cardiovascular control in hypertension-related left ventricular dysfunction. Hypertension 53: 205-209.

10. Grassi G, Cattaneo BM, Seravalle G, Lanfranchi A, Pozzi M, et al. (1997) 
Effects of chronic ACE inhibition on sympathetic nerve traffic and baroreflex control of circulation in heart failure. Circulation 96: 1173-1179.

11. Yuyama R, Yuasa F, Hikosaka M, Mimura J, Kawamura A, et al. (2005) lodine 123-metaiodobenzylguanidine imaging reflect generalized sympathetic activation in patients with left ventricular dysfunction. Clin Physiol Funct Imaging 25: 34-39.

12. Somsen GA, van Vlies B, de Milliano PA, Borm JJ, van Royen EA, et al. (1996) Increased myocardial [123I]-metaiodobenzylguanidine uptake after enalapril treatment in patients with chronic heart failure. Heart 76: 218-222.

13. Peach MJ, Cline WH, Jr., Watts DT (1966) Release of adrenal catecholamines by angiotensin. II. Circulation research 19: 571-575.

14. Comline RS, Silver M, Sinclair DG (1968) The effects of bradykinin angiotensin and acetylcholine on the bovine adrenal medulla. J Physiol 196 $339-350$

15. Raasch W, Betge S, Dendorfer A, Bartels T, Dominiak P (2001) Angiotensin converting enzyme inhibition improves cardiac neuronal uptake of noradrenaline in spontaneously hypertensive rats. J Hypertens 19: 18271833.

16. Dominiak P (1993) Modulation of sympathetic control by ACE inhibitors. Eur Heart J 14: 169-172.

17. Onishi T, Tanaka H, Yamadori Y, Shigeru M, Matsumoto K, et al. (2013) Left ventricular diastolic function is associated with cardiac sympathetic nerve activity in heart failure patients. Eur Heart $\mathrm{J} 34$

18. Dart AM, Du XJ, Kingwell BA (2002) Gender, sex hormones and autonomic nervous control of the cardiovascular system. Cardiovasc Res 53: 678-687.

19. Shoemaker JK, Norton KN, Baker J, Luchyshyn T (2015) Forebrain organization for autonomic cardiovascular control. Auton Neurosci 188: 5-9.

20. Iturriaga R, Del Rio R, Idiaquez J, Somers VK (2016) Carotid body chemoreceptors, sympathetic neural activation, and cardiometabolic disease. Biol Res 49: 13.

Copyright: (C) 2016 Abo-salem E, et al. This is an open-access article distributed under the terms of the Creative Commons Attribution License, which permits unrestricted use, distribution, and reproduction in any medium, provided the original author and source are credited. 